\title{
Can photoacoustic imaging quantify surface-localized J-aggregating nanoparticles?
}

\author{
Liang Lim \\ Robert Mastragostino \\ Kenneth Ng \\ Gang Zheng \\ Brian C. Wilson
}




\title{
Can photoacoustic imaging quantify surface-localized J-aggregating nanoparticles?
}

\author{
Liang Lim, ${ }^{a}$ Robert Mastragostino, ${ }^{a}$ Kenneth Ng, ${ }^{b}$ Gang Zheng, ${ }^{a, b}$ and Brian C. Wilson ${ }^{a, b, *}$ \\ aUniversity Health Network, Princess Margaret Cancer Centre, Toronto, Ontario, Canada \\ bUniversity of Toronto, Department of Medical Biophysics, Toronto, Ontario, Canada
}

\begin{abstract}
We investigate the feasibility of photoacoustic (PA) imaging to quantify the concentration of surfacelocalized nanoparticles, using tissue-mimicking phantoms and imaging with a commercial PA instrument at $815 \mathrm{~nm}$ and a linear-array transducer at a center frequency of $40 \mathrm{MHz}$. The nanoparticles were J-aggregating porphysomes (JNP) comprising self-assembling, all-organic porphyrin-lipid micelles with a molar absorption coefficient of $8.7 \times 10^{8} \mathrm{~cm}^{-1} \mathrm{M}^{-1}$ at this wavelength. The PA signal intensity versus JNP areal concentration followed a sigmoidal curve with a reproducible linear range of $\sim 17 \mathrm{fmol} / \mathrm{mm}^{2}$ to $11 \mathrm{pmol} / \mathrm{mm}^{2}$, i.e., $\sim 3$ orders of magnitude with $\pm 34 \%$ error. For physiologically-relevant conditions (i.e., optical scattering-dominated tissues: transport albedo $>0.8$ ) and JNP concentrations above $\sim 330 \mathrm{fmol} / \mathrm{mm}^{2}$, the PA signal depends only on the nanoparticle concentration. Otherwise, independent measurement of the optical absorption and scattering properties of the underlying tissue is required for accurate quantification. The implications for surface PA imaging, such as in the use of targeted nanoparticles applied topically to tissue as in endoscopic diagnosis, are considered. ๑ 2017 Society of Photo-Optical Instrumentation Engineers (SPIE) [DOI: 10.1117/1.JBO.22.7.076008]
\end{abstract}

Keywords: photoacoustic spectroscopy; nanophotonics; biomedical optics; optical properties.

Paper 170042R received Jan. 16, 2017; accepted for publication Jun. 21, 2017; published online Jul. 13, 2017.

\section{Introduction}

Photoacoustic (PA) imaging is a rapidly developing imaging technique, combining the molecular specificity of light with the deeper imaging capability of ultrasound. ${ }^{1}$ Depending on the platform, photoacoustic imaging (PAI) is capable of imaging optical absorbers at depths of multiple optical pathlengths (PA tomography) or with high resolution (PA microscopy). This positions PAI in a unique position for medical imaging applications, wherein the ability to image optical absorbers (e.g., blood, optically active nanoparticles) provides complementary information to structural imaging. ${ }^{2}$ Many groups have demonstrated PAI's potential for in vivo functional imaging, including mapping hemoglobin concentration and oxygen saturation, ${ }^{3,4}$ photosensitizing dyes, ${ }^{5}$ iron levels, ${ }^{6}$ and metal nanoparticles. ${ }^{7,8}$

Our recent development of porphysomes has added another dimension to PAI, enabling disease diagnosis, ${ }^{9}$ treatment monitoring, ${ }^{10}$ and real-time monitoring of nanoparticle distribution in vivo. ${ }^{10}$ Porphysomes are organic nanovesicles $(\sim 100 \mathrm{~nm})$ made from self-assembling porphyrin-lipid bilayers. ${ }^{9,10}$ They are photoacoustically active due to their extremely high and wavelength-tunable optical extinction $\left(\sim 3 \times 10^{9} \mathrm{~cm}^{-1} \mathrm{M}^{-1}\right.$ for pyropheophorbide porphysome at $680 \mathrm{~nm})$ that is comparable to that of gold nanoparticles. This same property makes them suitable to enhance and spatially localize energy deposition in photothermal therapy. ${ }^{9,10}$ Their porphyrin fluorescence and photodynamic activity are self-quenched in the intact state due to the high porphyrin concentration ( $\sim 80,000$ porphyrin molecules per nanoparticle), but become active upon cell uptake and disassembly into monomers. ${ }^{9,11}$ Porphysomes can be biomarker targeted, for example, to tumors by antibody labeling. ${ }^{9}$ PAI would then, in principle, be able to quantify the concentration of porphysomes

*Address all correspondence to: Brian C. Wilson, E-mail: wilson@uhnres. utoronto.ca in the target tumor in vivo, giving a measure of the biomarker expression level, which is becoming increasingly important for diagnosis, staging, and therapeutic guidance ${ }^{12-15}$ as, for example, in breast cancer to assess hormone receptors and epidermal growth factor receptor (EGFR). ${ }^{13}$

One example of the potential use of PAI is detection and localization of dysplasia in patients with Barrett's esophagus (BE) ${ }^{16}$ As the most important risk factor ( $>30$-fold) for esophageal adenocarcinoma, ${ }^{17} \mathrm{BE}$ is a condition in which the esophageal squamous mucosa (whitish in appearance on standard endoscopy) transforms to intestinal-type mucosa (pink-red appearance) due to chronic gastroesophageal reflux. BE is readily distinguishable from normal esophagus by endoscopy, but detection of dysplasia from nondysplastic BE is challenging under current standard endoscopic guidance. The current clinical standard is a four-quadrant random biopsy every 1 to $2 \mathrm{~cm}$ along the length of the Barrett's segment, leaving up to $99 \%$ of the tissue unsampled. Hence, there is a need for rapid and wide-field imaging for dysplasia diagnosis in BE. Many different optical techniques have been investigated to address this limitation, including diffuse reflectance spectroscopy, ${ }^{18}$ tissue autofluorescence, ${ }^{19}$ contrastbased fluorescence, ${ }^{20}$ Raman spectroscopy, ${ }^{21}$ optical coherence tomography, ${ }^{22,23}$ and narrowband imaging, ${ }^{24}$ but the challenge remains in detecting dysplasia in BE with high accuracy and to determine if there is submucosal spread of disease, which is critical in therapeutic decision-making. The potential advantages of PAI are its capability for rapid scanning of large tissue areas for detection, and imaging of the full esophageal thickness and beyond for staging. PAI devices suitable for esophageal endoscopy are under development. ${ }^{25}$ We are currently evaluating the feasibility and efficacy of intrinsic PAI (i.e., without any exogenous contrast agent), using ex vivo endoscopic mucosal

1083-3668/2017/\$25.00 @ 2017 SPIE 
resection tissues from BE-surveillance patients. ${ }^{16}$ While this has shown that PAI is indeed able to produce high-quality images of the tissue microvasculature, the initial findings suggest that specific contrast will be required to achieve high enough sensitivity and specificity. ${ }^{26}$ Hence, we hypothesize that combining PAI with targeted porphysomes will improve dysplasia detection in BE. Either systemic or topical administration of the porphysomes can be envisaged, the latter having the advantages of low total dose (minimizing cost and potential toxicity) and low background.

One of the major challenges in PAI is accurate quantification of the PA signal produced by physiologically relevant chromophore concentrations. As is well known, ${ }^{27}$ the PA signal can be written as

$p_{0}=\Gamma \mu_{a} \Phi$,

where $p_{0}$ is the initial acoustic pressure distribution, $\Gamma$ is the Grüneisen parameter (a tissue-dependent measure of the PA conversion efficiency), $\mu_{\mathrm{a}}$ is the chromophore's optical absorption (optical extinction coefficient multiplied by concentration), and $\Phi$ is the local light fluence. Solving for chromophore concentration from the PA signal is a difficult inverse problem due to these spatially varying parameters: $\Phi, \Gamma$, optical, and acoustic propagation. ${ }^{27,28}$ In addition, the PA signal is not uniform even from a homogenously absorbing tissue, but rather it is dominated by signals from tissue boundaries. ${ }^{29,30}$ Nevertheless, it is possible in principle to create a direct relationship between known chromophore concentration and PA intensity by strict control of the experimental conditions.

The purpose of the present study was to determine the feasibility and accuracy of photoacoustic tomography (PAT) in quantifying the areal concentration of topical porphysomes from the PA signal intensity, using tissue-mimicking optoacoustic phantoms as well-controlled models with varying optical properties at the PA wavelength. In PAT, the standard approach is to flood the field of view with pulsed laser light delivered to the tissue through fiber optic bundles integrated with the ultrasound transducer array. ${ }^{31}$ One can expect that the light fluence within tissue will not be homogenous and will attenuate with depth, depending on the tissue optical properties. In particular, the local light fluence experienced by surface-localized nanoparticles is the sum of the incident (primary) and backscattered (diffusely reflected) light. It is well established that the diffuse reflectance is a function of the transport albedo $\left(a^{\prime}\right)$ defined as $a^{\prime}=\mu_{s}^{\prime} /\left[\mu_{a}+\mu_{s}^{\prime}\right]$, where $\mu_{a}$ is the absorption coefficient and $\mu_{s}^{\prime}$ is the reduced scattering coefficient. ${ }^{32}$ Hence, measuring $a^{\prime}$ should enable correct normalization of the PA signal for the total light fluence experienced by the surface nanoparticles to calculate their areal concentration.

For the first part of this study, we measured the PA signal from varying concentrations of surface-localized porphysomes on tissue-mimicking phantoms comprising gelatin with known and varied absorption and scattering. The PA signal versus porphysomes concentration was plotted and the corresponding errors were calculated. We considered also how the phantom optical properties affect the PA signal.

\section{Materials and Methods}

\subsection{Photoacoustic System and Data Acquisition Procedure}

We used a commercial PAT imaging instrument (Vevo LAZR, Visualsonics, Toronto, Ontario, Canada), described in detail elsewhere. ${ }^{31}$ This instrument has an integrated fiber-optic and linear-array transducer (40 MHz center frequency, 256 elements) with a crossed-beam geometry for optical excitation. Ultrasound and PA images were collected at a frame rate of $5 \mathrm{~Hz}$ across a field of view of $\sim 15 \mathrm{~mm}$ (depth) by $14 \mathrm{~mm}$ (width). The transducer was mounted on a motorized translational stage to scan over a three-dimensional (3-D) volume, and PA images were acquired in $0.1-\mathrm{mm}$ increments at $815 \mathrm{~nm}$. Due to the crossed laser beam geometry of the PAI transducer probe, ${ }^{31}$ there is an optimal PA zone within the field of view at $\sim 9$ to $11 \mathrm{~mm}$ from the probe surface. For consistency in the transducer orientation and incident light fluence $\left(\Phi_{0}\right)$, we positioned the surface of the phantom $9 \mathrm{~mm}$ from the transducer for all measurements.

\subsection{Sample Preparation}

J-aggregate porphysomes (JNP) comprising bacteriopheophorbide a-lipid dye was used, as described elsewhere. ${ }^{10}$ These are of particular interest for PA monitoring and enhancement of photothermal therapy, where quantification is important as part of dose optimization. ${ }^{10}$ They have typical porphysome optical absorption spectra, as shown in the insert of Fig. 1. To simulate topically applied nanoparticles on tissue, we used JNP on the surface of tissue-mimicking phantoms made from gelatin and Intralipid, ${ }^{33}$ as shown in Fig. 1 . The procedure was to pipette $50 \mu \mathrm{l}$ of varying concentrations $(0.355 \mathrm{nM}$ to $118 \mu \mathrm{M})$ of JNPs in phosphate-buffered serum (PBS) onto the surface [Fig. 1(a)] and leave it overnight at room temperature to evaporate [Fig. 1(b)] while protected from light by aluminum foil to prevent photobleaching. A thin $(\sim 0.5 \mathrm{~mm})$ layer of clear gelatin was added on top [Fig. 1(c)] to avoid physical damage by the PAI probe and ultrasound coupling gel during imaging.

In the main experiments, the goal was to measure the PA signal as a function of both the JNP concentration and optical properties of the tissue-mimicking phantom base. Based on published data for normal esophageal tissue $\left(\mu_{a}=0.080 \pm\right.$ $0.023 \mathrm{~mm}^{-1}$ and $\mu_{s}^{\prime}=0.77 \pm 0.15 \mathrm{~mm}^{-1}$ at $\left.630 \mathrm{~nm}\right),{ }^{34}$ we selected the range $a^{\prime}=0.65$ to 0.95 to represent the expected maximum range of esophageal tissue, as relevant to ongoing PAI studies in BE patients, ${ }^{12}$ while recognizing that the variability in Barrett's tissue is likely much larger than that in normal squamous esophagus because of the high inflammatory component. ${ }^{16}$ The phantom bases were created using 5\% weight/ volume gelatin (GX50, Matheson Coleman \& Bell, Norwood, Ohio) in reverse-osmosis water, with intralipid (Fresenius Kabi, Richmond Hill, Ontario, Canada) added at different concentrations to achieve the desired $\mu_{s}^{\prime}$. The optical properties of intralipid were experimentally verified using the added-absorber technique. ${ }^{35}$ Our experimental results show up to $40 \%$ reduction in optical scattering for the same intralipid concentration in water compared with the value in gelatin, consistent with the findings of Cook et al. ${ }^{33}$ However, the impact on $a^{\prime}$ is less marked. We created tissue-mimicking phantom bases with $a^{\prime}$ spanning the targeted range, with optical properties varied as per Table 1. The intrinsic absorption of intralipid is negligible $\left(<0.001 \mathrm{~mm}^{-1}\right)$ compared with that of $5 \%$ gelatin $\left(0.051 \mathrm{~mm}^{-1}\right.$, measured by an UV-vis spectrophotometer).

The dilution of JNP spanned five orders of magnitude $(0.0011 \%$ to $33 \%)$ by diluting from the stock $355-\mu \mathrm{M}$ concentration. The JNP concentration after deposition is defined in $\mathrm{pmol} / \mathrm{mm}^{2}$ based on the known concentration of the drops before drying, indicating the near monolayer geometry and averaging 
(a)

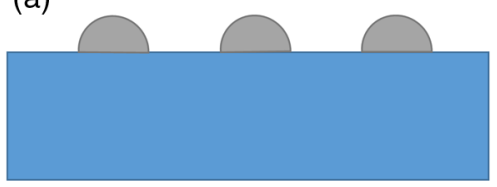

(d)

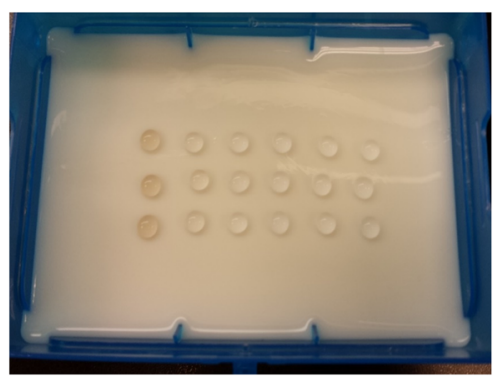

(b)

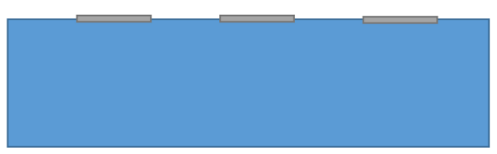

(e)

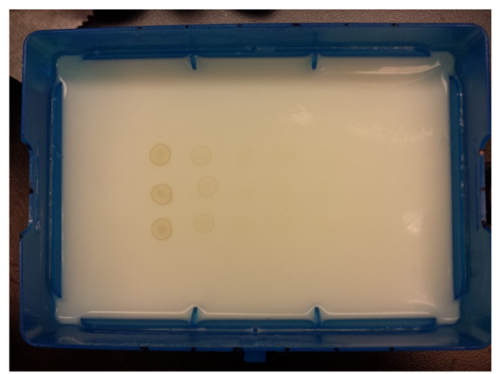

(c)

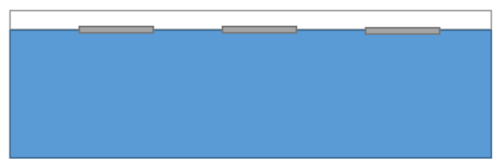

(f)

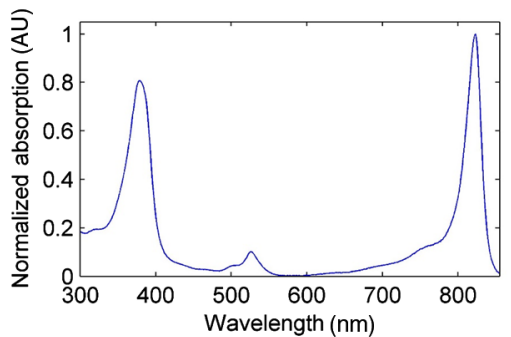

Fig. 1 Schematic and photographs of the steps in preparing the topical-JNP phantoms. (a, d) 50- $\mu$ l droplets of known concentrations of JNP on tissue-mimicking phantom base. (b) After evaporation. (c, e) After depositing a thin layer of clear gelatin. Each column of spots represents three replicates of JNP droplets in decreasing order of concentration. ( $\mathrm{d}$ and $\mathrm{e}$ ) are photographs using the highest scattering phantom base. (f) Optical absorption spectra (normalized) of JNP in PBS.

Table 1 Optical properties of the phantom base at $815 \mathrm{~nm}$, close to the JNP absorption peak at $824 \mathrm{~nm}$.

\begin{tabular}{lcccc}
$a^{\prime}$ & $\mu_{s}^{\prime}\left(\mathrm{mm}^{-1}\right)$ & $\mu_{a}\left(\mathrm{~mm}^{-1}\right)$ & Water $(\mathrm{ml})$ & $\begin{array}{c}\text { Intralipid } \\
20 \%(\mathrm{ml})\end{array}$ \\
\hline Zero (0) & 0 & 0.051 & 250 & 0 \\
$\begin{array}{l}\text { Low }(0.65 \\
\text { to 0.76) }\end{array}$ & 0.095 to 0.158 & 247 & 3 \\
$\begin{array}{l}\text { Medium }(0.81 \\
\text { to 0.88) }\end{array}$ & 0.220 to 0.367 & & \\
$\begin{array}{l}\text { High }(0.9 \\
\text { to 0.95) }\end{array}$ & 0.439 to 0.733 & 244.4 & 5.6 \\
\hline
\end{tabular}

over the drop size because there were unavoidable variations from drop to drop in the JNP distribution after drying.

\subsection{Data Analysis}

PAI and ultrasound images were analyzed in MATLAB (Mathworks, Natick, Massachusetts), as shown in Fig. 2. A logarithmic scale $\left[20 \log _{10}(\mathrm{~dB})\right]$ was applied to the onboard beamformed PA signals. From the original 3-D volume [Fig. 2(a)], the PA signal was flattened to two-dimensions [Fig. 2(b)] by isolating the maximum intensity of each column perpendicular to the tissue surface. A PA intensity threshold was applied to this, and the JNP droplet was identified by visual inspection. We then applied an area threshold $\left(16.5 \mathrm{~mm}^{2}\right.$, i.e., $<\sim 50 \%$ of the droplet area) to exclude the surrounding discrete spots that, we believe, were due to tiny air bubbles trapped between the phantom layers during fabrication. The air bubbles are less visible within the JNP droplet area, likely due to their relatively low PA intensity. The PA values within the resulting area [Fig. 2(c)] were averaged to represent the PA signal for that JNP areal concentration.

\section{Results}

Figure 3 plots the relationship between PA signal and JNP areal concentration ( $\mathrm{pmol} / \mathrm{mm}^{2}$ ) measured for the phantoms of different $a^{\prime}$. The solid lines are best sigmoid fits of the form $\mathrm{PA}=\frac{a}{1+\exp [-b(x+d)]}+c$, where $x$ is $\log _{10}$ (JNP areal concentration) and $a, b, c$ are variables in the fit. All the curves converged at $\sim 82 \mathrm{~dB}$ as the concentration increased, consistent with PA measurement of a high concentration of India ink (1\%) shown by the black dashed horizontal line. The noise floor of the PAI system is at $\sim 29 \mathrm{~dB}$.

As expected qualitatively, the PA intensity increased monotonically and consistently with increasing JNP concentration and phantom albedo, confirming that there is a significant contribution to the PA signal from light that is diffusely backscattered to the surface. The increase and leftshifting of the floor of the sigmoid curves with increasing $a^{\prime}$ are consistent with this interpretation and allows detection of lower JNP concentrations. For physiologically relevant conditions (i.e., optical scatteringdominated tissues: transport albedo $>0.8$ ) and JNP concentrations above $\sim 330 \mathrm{fmol} / \mathrm{mm}^{2}$, the PA signal depends only on the nanoparticle concentration (note the sigmoids for medium and high $a^{\prime}$ values are practically indistinguishable above $330 \mathrm{fmol} /$ $\mathrm{mm}^{2}$ ). In the (biologically unlikely) case of very high JNP areal concentration, the PA signal becomes essentially independent of concentration, i.e., the signal saturates.

We defined the concentration estimate error as the difference between the sigmoid fit and its $95 \%$ confidence intervals, as shown in Fig. 4. Using medium $a^{\prime}$ as an example, we first calculated the confidence intervals [Fig. 4(a)], shown as the dashed lines to the left and right of the sigmoid. These are smallest in the linear portion of the signal response curves and increase as the curves flatten out at both ends. We then calculated the error for each JNP areal concentration. Using medium $a^{\prime}$ as an example [Fig. 4(b)], a PA value of $39 \mathrm{~dB}$ corresponded to $0.017 \mathrm{pmol} /$ $\mathrm{mm}^{2}$ with confidence intervals of 0.0102 to $0.0219 \mathrm{pmol} / \mathrm{mm}^{2}$. The errors between the fit and confidence intervals were $29 \%$ $(0.0219 / 0.017$ to 1$)$ and $40 \%$ ( 1 to $0.0098 / 0.015)$, resulting in a mean error of $\pm 34 \%$ at $0.017 \mathrm{pmol} / \mathrm{mm}^{2}$ [Fig. 4(c)]. In 

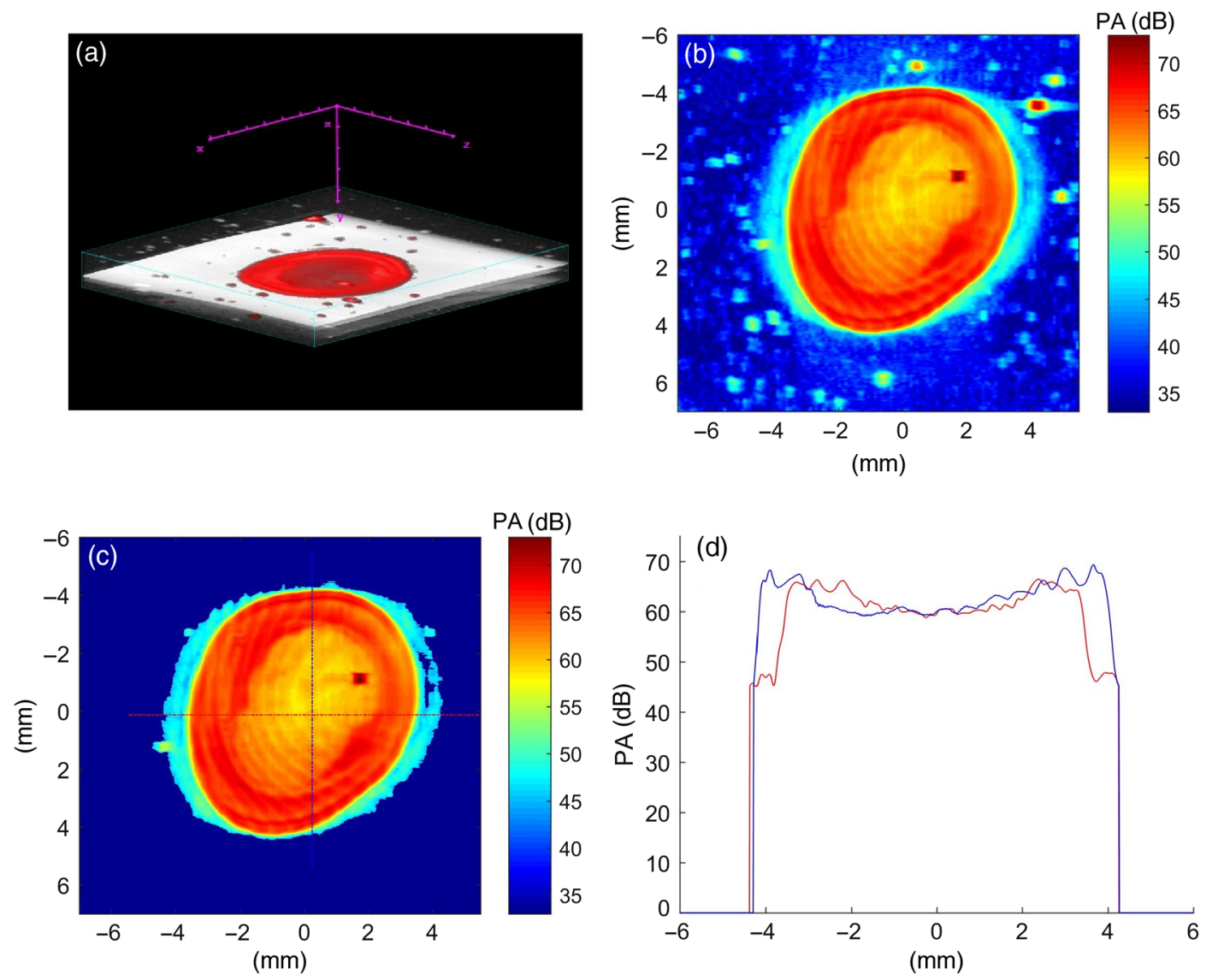

Fig. 2 Example of the PAI data analysis procedure taken from one of the three replicates for $0.06 \%$ JNP concentration on the phantom base with high transport albedo. (a) 3-D reconstruction with the PA signal in red overlaid on the grayscale ultrasound image. (b) 2-D surface flattened from the 3-D volume: color scale bar in dB. (c) JNP droplet area isolated after PA intensity and area thresholding: the blue and red dotted lines show the locations of the profiles plotted in (d).

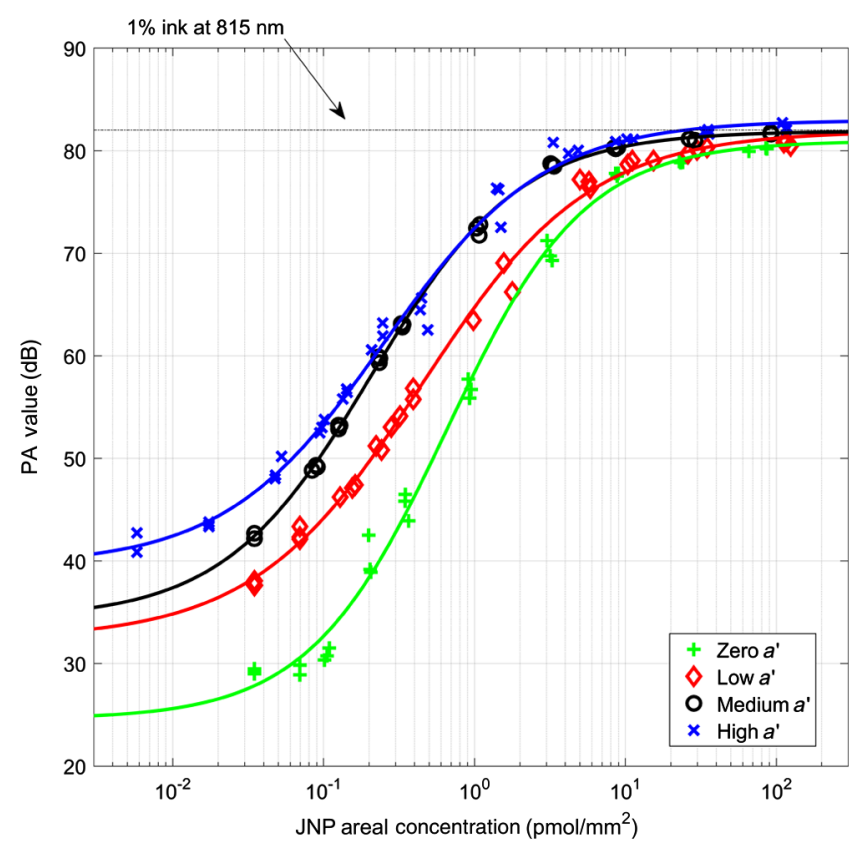

Fig. 3 PA intensity $(\mathrm{dB})$ at $815 \mathrm{~nm}$ versus JNP areal concentration for $a^{\prime}$ values in the phantom base. The solid lines represent sigmoidal fits to the measurements. The black dashed line (PA $=82 \mathrm{~dB}$ for $1 \%$ India ink) indicates the maximum PA signal level of the instrument. general, at a maximum error of $\pm 34 \%$ for zero, low, and high $a^{\prime}$ values, we can quantify the JNP areal concentration within 1 to 2 orders of magnitude (Appendix). This range is extended to almost 3 orders of magnitude $\left(0.017\right.$ to $\left.11 \mathrm{pmol} / \mathrm{mm}^{2}\right)$ for medium $a^{\prime}$.

To further understand and confirm the increase and shift of sigmoid curves, we observed in Fig. 3, we created multiple tissue-mimicking phantom bases with different optical properties and measured the PA signal generated by each without added porphysomes. We found that the PA signal correlated best to the product of the $\mu_{a}$ and $a^{\prime}$, as shown in Fig. 5.

\section{Discussion and Conclusions}

Our goal was to establish the relationship between the PA signal from the surface nanoparticles and their areal concentration using PAT. Given a measured PA value, we can use Figs. 3 and 4(c) to estimate the JNP areal concentration \pm error, respectively. For instance, for a JNP PA signal of $65 \mathrm{~dB}$ on medium- $a^{\prime}$ tissue, the predicted areal concentration is $0.407 \mathrm{pmol} / \mathrm{mm}^{2} \pm$ $4.6 \%$. The quantifiable range depends on the required accuracy of the measurement. For example, for a maximum error of $\pm 34 \%$ ( 1 standard deviation of a normal distribution) on medium- $a^{\prime}$ tissue, the lowest and highest quantifiable JNP concentrations are 0.017 and $11 \mathrm{pmol} / \mathrm{mm}^{2}$, respectively. To estimate the JNP concentration more accurately, knowledge of the underlying tissue's optical properties may be required, in 

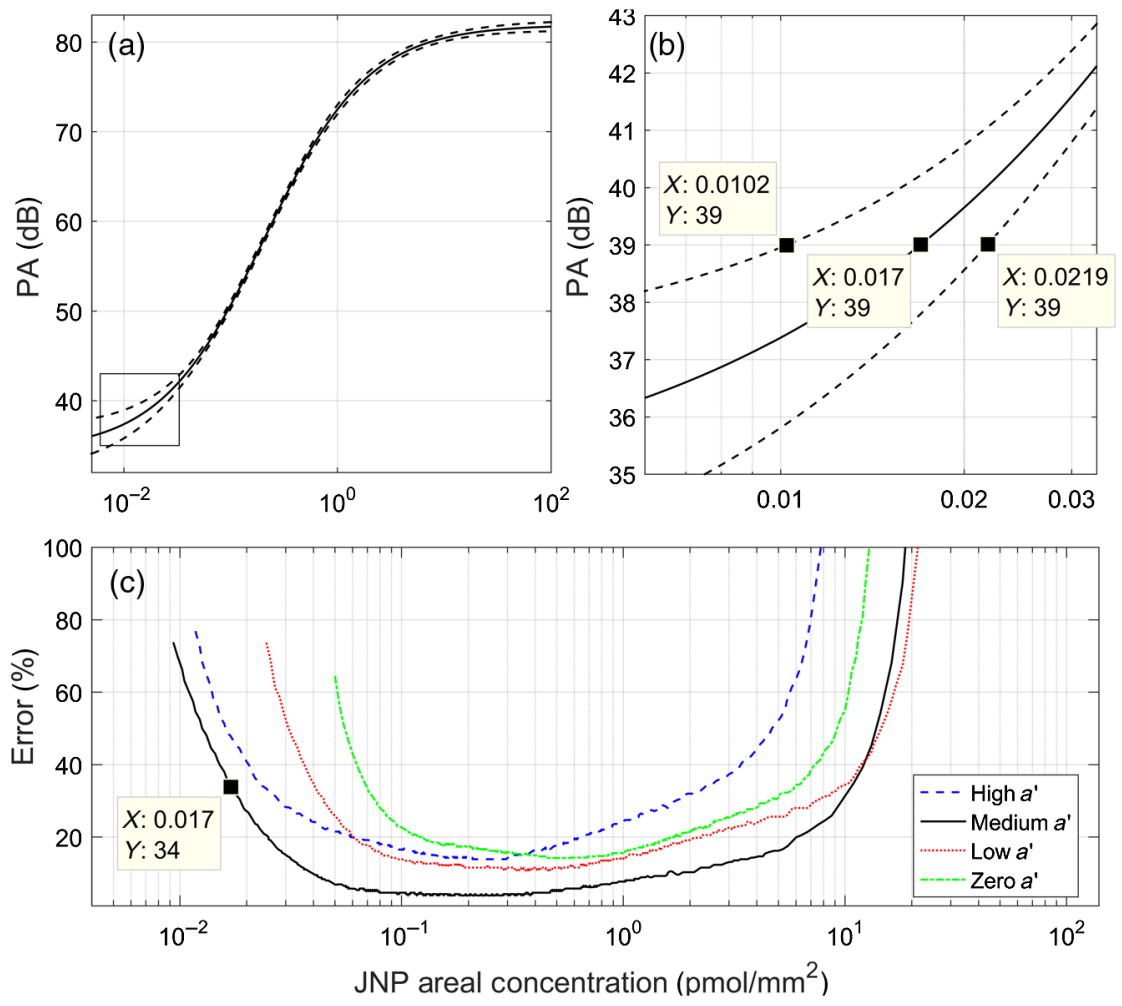

Fig. 4 Error in estimating JNP areal concentration. (a) 95\% confidence intervals (dashed lines) of medium-a' sigmoid. (b) Magnified plot of the box in a. (c) Error versus JNP areal concentration plot for all sigmoids. All $x$-axis are JNP areal concentration $\left(\mathrm{pmol} / \mathrm{mm}^{2}\right)$.

particular when the PA signal from the nanoparticles is low $(<\sim 60 \mathrm{~dB})$ or when the transport albedo of the tissue is low. These optical properties could be measured in vivo by, for example, diffuse reflectance measurements. ${ }^{18,36}$

The increase in the PA signal with increasing JNP concentration is, of course, as would be expected qualitatively from
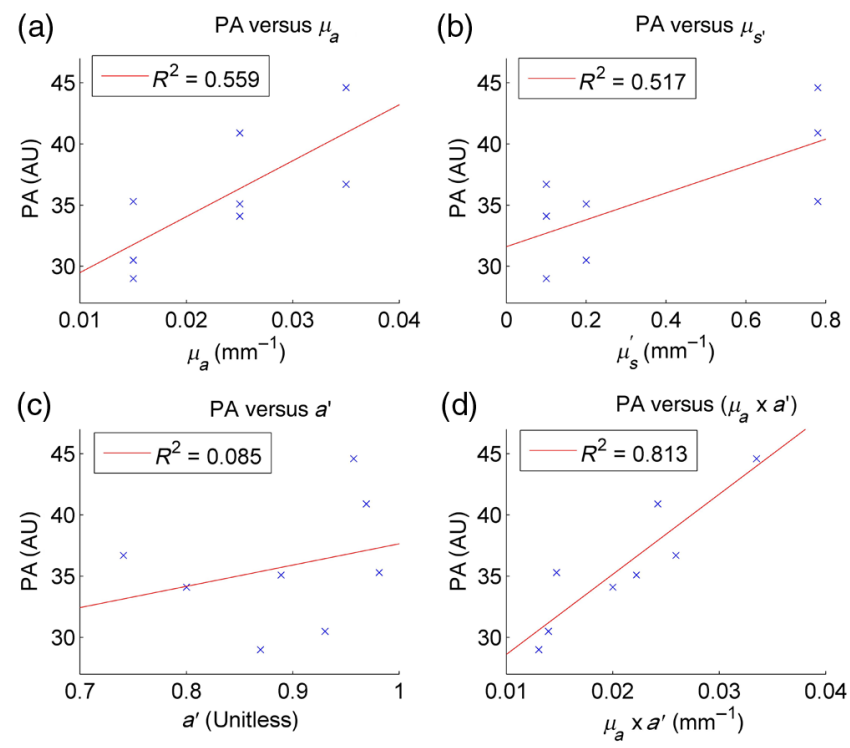

Fig. 5 Background PA signal versus optical properties of the phantom base, together with best linear fits. Note the significant scatter in the measurements due to difference in optical properties. The best correlation is with the product of $\mu_{a}$ and $a^{\prime}(\mathrm{d})$.
Eq. (1), holding $\Phi$ and $\Gamma$ as constant while increasing the optical absorption. The S-shaped response curves of PA versus JNP concentrations show a central linear region with the detector noise floor and ceiling, typical of sensor response curves. ${ }^{37} \mathrm{~A}$ sigmoid was used as the simplest equation to fit data of this form, as for example in drug dose-response curves. ${ }^{38}$ The sigmoid-form relationship between PA versus JNP areal concentration should be universal for surface-localized chromophores. We also highlight the general shape of the quantitation error, as shown by the plot in Fig. 4(c). Quantitation error is lower at the center of the sigmoids $\left(\sim 10^{-1}\right.$ to $\left.10^{0} \mathrm{pmol} / \mathrm{mm}^{2}\right)$, and error increases as JNP concentration approaches the floor or ceiling of the sigmoids. This is expected as the floor and ceiling of the sigmoids are at the edge of the PA system's dynamic range. PA signal contrast between different concentrations is reduced, resulting in higher quantification error.

As previously mentioned, in this experimental geometry, where PAT uses a pulsed laser to flood the field of view from the top, the fluence attenuates with depth depending on the tissue optical scattering and absorption coefficients. Higher scattering also results in higher backscattered (diffusely reflected) light reaching the tissue surface. The local light fluence $(\Phi)$ on the tissue surface is

$\Phi=\Phi_{o}+\Phi_{R}$

where $\Phi_{o}$ is from the incident (primary) light and $\Phi_{R}$ is the back-scattered light (which depends on $a^{132}$ ). The increase and leftshifting of the floor of the curves were found to correlate best with the product of the absorption and $a^{\prime}$ of the underlying phantom, as seen in Fig. 5. 
These results pose a question regarding the clinical translation feasibility of nanoparticle-contrast PA endoscopy, namely can a high enough surface density of biomarker-targeted topical porphysomes be achieved under typical in vivo conditions to give a reliable PAI signal intensity from which the porphysome surface concentration can be calculated? Estimating the binding affinity of targeted porphysomes is beyond the scope of this paper, but if the reasonable detectable JNP concentration we reported is orders of magnitude higher than the ideal case, where all tissue surface is bound to porphysome, then PAI is not likely to be a good platform for quantitative porphysome imaging. Assuming that the accessible tissue surface of interest (e.g., dysplasia or carcinoma in situ) is completely covered with porphysomes bound to cell-surface receptors and tightly packed, then the areal concentration would be $\sim 10^{8} \mathrm{JNP} / \mathrm{mm}^{2}$. At $\pm 34 \%$ error, the lowest quantifiable concentration is $\sim 7.81$ to $31.8 \times 10^{5} \mathrm{JNP} / \mathrm{mm}^{2}$ (Appendix), at least 2 orders of magnitude lower than the ideal case of $10^{8} \mathrm{JNP} / \mathrm{mm}^{2}$. Hence, PAI detection and quantification of surface-bound targeted porphysomes on tissue seems feasible.

These results pose another question: how well do our phantoms match general antibody-based tissue surface labeling? The distribution of JNP within our phantom spots is relatively uniform, as can be seen from the line profiles of a typical spot in Fig. 2(d). Using EGFR as an example, in vitro studies have shown uniform distribution of cell-surface receptors ${ }^{39,40}$ and anti-EGFR antibody conjugated gold nanoparticles bound on cancer cell-surface receptors. ${ }^{41}$ But it is unlikely for cell-surface receptors/nanoparticles to remain uniformly distributed throughout cancerous tissue, where there may be multiple cell types at different disease stages. Nevertheless, we should still be able to see JNP PA hotspots above the minimal quantifiable threshold, quantify the JNP areal concentration, make relative comparison of biomarker expression level between disease stages and potentially estimate absolute biomarker expression level. These are subjects of ongoing studies and will be experimentally investigated in the near future, initially in ex vivo human endoscopic mucosal resection specimens ${ }^{26}$ with topically applied antibodyconjugated porphysomes. PA quantification of other porphysomes applications (e.g., subsurface/porphysomes within tumor, porphysomes within blood vessels through systemic delivery) is also subject of ongoing studies.

We are mindful of the limitations of these results. Our goal here was to investigate PA nanoparticle quantification for the particular situation ${ }^{28}$ of topically applied nanoparticles using PAT, so the conclusions are restricted to this scenario. The specific values of, e.g., the minimum detectible nanoparticle concentration, will also be particular to the experimental parameters used, such as the laser power and transducer sensitivity and the nanoparticle (i.e., JNP). In addition, an underlying assumption is that $\Gamma$ does not change with JNP concentration, which is reasonable as PA increases linearly with JNP concentration. It is unlikely for $\Gamma$ to change within the linear range or at low JNP concentration, but it is possible at high JNP concentration $\Gamma$ may not remain constant, and contribute to the rate of PA signal flattening. Nevertheless, the results do indicate that topical nanoparticle quantification should be feasible with a sensitivity and accuracy that would be relevant to clinical applications, such as porphysome contrast-enhanced endoscopy.

In conclusion, PAT can quantify surface-localized JNPs. Our results enable quantification of the porphysome areal density, and the quantifiable range is determined by the required accuracy. At
Table 2 Lowest JNP concentration with 34\% error.

\begin{tabular}{lccc}
\hline$a^{\prime}$ & $\mathrm{pmol} / \mathrm{mm}^{2}$ & $\mathrm{bchl} / \mathrm{mm}^{2}$ & $\mathrm{JNP} / \mathrm{mm}^{2}$ \\
\hline 0 & 0.0692 & $4.17 \times 10^{10}$ & $3.18 \times 10^{6}$ \\
Low & 0.0407 & $2.45 \times 10^{10}$ & $1.87 \times 10^{6}$ \\
Medium & 0.017 & $1.02 \times 10^{10}$ & $7.81 \times 10^{5}$ \\
High & 0.0234 & $1.41 \times 10^{10}$ & $1.08 \times 10^{6}$ \\
\hline
\end{tabular}

Table 3 Highest JNP concentration with 34\% error.

\begin{tabular}{lccc}
$a^{\prime}$ & $\mathrm{pmol} / \mathrm{mm}^{2}$ & $\mathrm{bchl} / \mathrm{mm}^{2}$ & $\mathrm{JNP} / \mathrm{mm}^{2}$ \\
\hline 0 & 5.89 & $3.55 \times 10^{12}$ & $2.71 \times 10^{8}$ \\
Low & 9.77 & $5.88 \times 10^{12}$ & $4.49 \times 10^{8}$ \\
Medium & 11 & $6.62 \times 10^{12}$ & $5.05 \times 10^{8}$ \\
High & 2.34 & $1.41 \times 10^{12}$ & $1.08 \times 10^{8}$ \\
\hline
\end{tabular}

$\pm 34 \%$ error on medium- $a^{\prime}$ tissue, the quantifiable areal concentration is in the range of $17 \mathrm{fmol} / \mathrm{mm}^{2}$ to $11 \mathrm{pmol} / \mathrm{mm}^{2}$, which should be sufficient for clinical applications, such as biomarkerbased diagnosis, or guiding subsequent ablative therapies, such as porphysome-enhanced photothermal therapy. ${ }^{9}$ In correcting the measured signal for the effects of backscattered light from the tissue, we need to know the tissue optical properties, which can be measured from the diffuse reflectance. For endoscopic applications, one can then envisage ways to integrate both PAI and diffuse reflectance measurements in the same probe.

\section{Appendix: Estimate of Number of J-Aggregating Porphysomes Per Square Millimeter Surface Area}

For porphysomes, the molar concentration refers to the concentration of the component porphyrin-lipid conjugate, which is bacteriochlorophyll (bchl) molecule in the case of JNP. There are $\sim 83,000$ porphyrin-lipid conjugates per 100-nm diameter JNP containing 95\% porphyrin-lipid ${ }^{9}$ and with $15 \mathrm{~mol} \%$ of bchl in the JNP's formulation. ${ }^{10}$ This gives:

Number of bchl molecules per JNP nanoparticle $=$ $15 / 95 \times 83000=13105$

Thus

$$
\begin{aligned}
0.195 \mathrm{pmol} / \mathrm{mm}^{2} & =0.195 \times 10^{-12} \mathrm{~mol} / \mathrm{mm}^{2} \\
& =0.195 \times 10^{-2} \times 6.022 \times 10^{23} \mathrm{bchl} / \mathrm{mm}^{2} \\
& =1.17 \times 10^{11} / 13105 \mathrm{JNP} / \mathrm{mm}^{2} \\
& =8.96 \times 10^{6} \mathrm{JNP} / \mathrm{mm}^{2}
\end{aligned}
$$

For each $a^{\prime}$, the lowest and highest quantifiable JNP concentrations at $\pm 34 \%$ error are shown in Tables 2 and 3, respectively.

\section{Disclosures}

The authors have no relevant financial interests in the paper and no other potential conflicts of interest to disclose. 


\section{Acknowledgments}

This work was supported by an Emerging Frontiers grant (\#1022) from the Terry Fox Research Institute, Canada.

\section{References}

1. L. V. Wang, "Ultrasound-mediated biophotonic imaging: a review of acousto-optical tomography and photo-acoustic tomography," Dis. Markers 19(2-3), 123-138 (2004).

2. M. Xu and L. V. Wang, "Photoacoustic imaging in biomedicine," Rev. Sci. Instrum. 77(4), 041101 (2006).

3. X. Wang et al., "Noninvasive imaging of hemoglobin concentration and oxygenation in the rat brain using high-resolution photoacoustic tomography," J. Biomed. Opt. 11(2), 024015 (2006).

4. M. Sivaramakrishnan et al., "Limitations of quantitative photoacoustic measurements of blood oxygenation in small vessels," Phys. Med. Biol. 52(5), 1349-1361 (2007).

5. C. J. H. Ho et al., "Multifunctional photosensitizer-based contrast agents for photoacoustic imaging," Sci. Rep. 4, 5342 (2014).

6. F. Maccarinelli et al., "Photoacoustic molecular imaging for in vivo liver iron quantitation," J. Biomed. Opt. 21(5), 056008 (2016).

7. S. Mallidi et al., "Molecular specific optoacoustic imaging with plasmonic nanoparticles," Opt. Express 15(11), 6583-6588 (2007).

8. Y.-S. Chen et al., "Enhanced thermal stability of silica-coated gold nanorods for photoacoustic imaging and image-guided therapy," Opt. Express 18(9), 8867-8878 (2010).

9. J. F. Lovell et al., "Porphysome nanovesicles generated by porphyrin bilayers for use as multimodal biophotonic contrast agents," Nat. Mater. 10(4), 324-332 (2011).

10. K. K. Ng et al., "Stimuli-responsive photoacoustic nanoswitch for in vivo sensing applications," ACS Nano 8(8), 8363-8373 (2014).

11. M. Shakiba et al., "Stable J-aggregation enabled dual photoacoustic and fluorescence nanoparticles for intraoperative cancer imaging," Nanoscale 8(25), 12618-12625 (2016).

12. M. E. C. Bruno et al., "Correlation of biomarker expression in colonic mucosa with disease phenotype in Crohn's disease and ulcerative colitis," Dig. Dis. Sci. 60(10), 2976-2984 (2015).

13. E. A. Rakha, J. S. Reis-Filho, and I. O. Ellis, "Combinatorial biomarker expression in breast cancer," Breast Cancer Res. Treat. 120(2), 293-308 (2010).

14. C. Curtis et al., "The genomic and transcriptomic architecture of 2,000 breast tumours reveals novel subgroups," Nature 486(7403), 346-352 (2012).

15. J. Speiser et al., "Notch-1 and Notch-4 biomarker expression in triplenegative breast cancer," Int. J. Surg. Pathol. 20(2), 137-143 (2012).

16. L. Lim et al., "Clinical study of ex vivo photoacoustic imaging in endoscopic mucosal resection tissues," Proc. SPIE 9323, 932307 (2015).

17. M. Solaymani-Dodaran et al., "Risk of oesophageal cancer in Barrett's oesophagus and gastro-oesophageal reflux," Gut 53(8), 1070-1074 (2004).

18. A. Douplik et al., "Diffuse reflectance spectroscopy in Barrett's esophagus: developing a large field-of-view screening method discriminating dysplasia from metaplasia," J. Biophotonics 7(5), 304-311 (2014).

19. M. Kara et al., "Autofluorescence-based detection of early neoplasia in patients with Barrett's esophagus," Dig. Dis. 22(2), 134-141 (2004).

20. H. Messmann et al., "Fluorescence endoscopy for the detection of low and high grade dysplasia in ulcerative colitis using systemic or local 5aminolaevulinic acid sensitisation," Gut 52, 1003-1007 (2003).

21. M. S. Bergholt et al., "In vivo diagnosis of esophageal cancer using image-guided Raman endoscopy and biomolecular modeling," Technol. Cancer Res. Treat. 10(2), 103-112 (2011).

22. V. X. D. Yang et al., "Endoscopic Doppler optical coherence tomography in the human GI tract: initial experience," Gastrointest. Endosc. 61(7), 879-890 (2005).
23. B. Bouma and G. Tearney, "High-resolution imaging of the human esophagus and stomach in vivo using optical coherence tomography," Gastrointest. Endosc. 51(4), 467-474 (2000).

24. R. Singh et al., "Preliminary feasibility study using a novel narrow-band imaging system with dual focus magnification capability in Barrett's esophagus: is the time ripe to abandon random biopsies?" Dig. Endosc. 25, 151-156 (2013).

25. J.-M. Yang et al., "Catheter-based photoacoustic endoscope," J. Biomed. Opt. 19(6), 066001 (2014).

26. L. Lim et al., "A feasibility study of photoacoustic imaging of ex vivo endoscopic mucosal resection tissues from Barrett's esophagus patients," Endosc. Int. Open (2017), in press.

27. B. Cox et al., "Quantitative spectroscopic photoacoustic imaging: a review," J. Biomed. Opt. 17, 061202 (2012).

28. B. Cox, J. Laufer, and P. Beard, "The challenges for quantitative photoacoustic imaging," Proc. SPIE 7177, 717713 (2009).

29. Z. Guo, L. Li, and L. V. Wang, "On the speckle-free nature of photoacoustic tomography," Med. Phys. 36(9), 4084-4088 (2009).

30. R. K. Saha et al., "Validity of a theoretical model to examine blood oxygenation dependent optoacoustics," J. Biomed. Opt. 17(5), 055002 (2012).

31. A. Needles et al., "Development and initial application of a fully integrated photoacoustic micro-ultrasound system," IEEE Trans. Ultrason. Ferroelectr. Freq. Control 60(5), 888-897 (2013).

32. T. J. Farrell, M. S. Patterson, and B. C. Wilson, "A diffusion theory model of spatially resolved, steady-state diffuse reflectance for the noninvasive determination of tissue optical properties in vivo," Med. Phys. 19(4), 879-888 (1992).

33. J. R. Cook, R. R. Bouchard, and S. Y. Emelianov, "Tissue-mimicking phantoms for photoacoustic and ultrasonic imaging," Biomed. Opt. Express 2(11), 3193-3206 (2011).

34. P. R. Bargo et al., "In vivo determination of optical properties of normal and tumor tissue with white light reflectance and an empirical light transport model during endoscopy," J. Biomed. Opt. 10(3), 034018 (2005).

35. A. J. Welch and M. J. C. Van Gemert, Optical-Thermal Response of Laser-Irradiated Tissue, 2nd ed., Springer, Heidelberg (2011).

36. N. Rajaram et al., "Design and validation of a clinical instrument for spectral diagnosis of cutaneous malignancy," Appl. Opt. 49(2), 142152 (2010).

37. J. Janata, Principles of Chemical Sensors, Springer, New York (2010).

38. A. DeLean, P. J. Munson, and D. Rodbard, "Simultaneous analysis of families of sigmoidal curves: application to bioassay, radioligand assay, and physiological dose-response curves," Am. J. Physiol. 235(2), G97G102 (1978).

39. J. L. Boerner et al., "Phosphorylation of Y845 on the epidermal growth factor receptor mediates binding to the mitochondrial protein cytochrome c oxidase subunit II," Mol. Cell. Biol. 24(16), 7059-7071 (2004).

40. M. Bailly et al., "Epidermal growth factor receptor distribution during chemotactic responses," Mol. Biol. Cell. 11(11), 3873-3883 (2000).

41. I. H. El-Sayed, X. Huang, and M. A. El-Sayed, "Surface plasmon resonance scattering and absorption of anti-EGFR antibody conjugated gold nanoparticles in cancer diagnostics: applications in oral cancer," Nano Lett. 5(5), 829-834 (2005).

Liang Lim received his BS degree in electrical engineering and his $\mathrm{PhD}$ in biomedical engineering from the University of Texas at Austin in 2004 and 2013, respectively. He is a postdoctoral fellow at Princess Margaret Cancer Centre, University Health Network, Toronto, Ontario, Canada. His current research interests include biophotonics for disease diagnosis and therapy. He is a member of SPIE.

Biographies for the other authors are not available. 